\title{
CONTINUOUS FUNCTIONS ON EXTREMALLY DISCONNECTED SPACES
}

\author{
J. VERMEER
}

\begin{abstract}
Using results and techniques due to Abramovich, Arenson and Kitover it is shown that each fixed-point set of a selfmap of a compact extremally disconnected space is a retract of that space, and that the retraction can be constructed from the particular selfmap itself. Also, the closure of the set of periodic points turns out to be a retract of the space. Several decomposition theorems for arbitrary selfmaps on extremally disconnected spaces are obtained similar to the theorem of Frolík on embeddings. Conditions are obtained under which the set of fixed points is clopen.
\end{abstract}

\section{INTRODUCTION}

At first glance the class of compact extremally disconnected spaces might seem unworkable. However, there are many indications that the (continuous) selfmaps on spaces of this type behave 'extremally' well. On the 'extremally' nice space $[0,1]$ the quadratic familly shows that here the selfmaps might seem to be unworkable. So, it is a matter of taste or interest what to investigate. However, if one is only interested in the algebraic semigroup structure of the selfmaps, then there is reason to investigate the compact extremally disconnected spaces. Each such semigroup can be studied as a sub-semigroup of the semigroup of (even) open selfmaps of some compact extremally disconnected space.

So, topological dynamics is a field of mathematics where the extremally disconnected spaces appears. Another field is the operator theory. In fact, the basic ideas and constructions used in this paper are from results in this field of mathematics, and are not very well known by topologists. In particular, the work of A.K. Kitover deserves more attention. Several results and ideas are from him, see $\left[K_{1}\right]$ and $[A, A, K]$. But the results were less explicit and incorporated in the analysis of a somewhat less general situation.

The theorem of Frolik is the classical result of the good behaviour of maps on compact extremally disconnected spaces:

"The fixed-point set of every selfembedding of a compact extremally disconnected space is clopen"

Received by the editors March 2, 1993 and, in revised form, January 27, 1994. 1991 Mathematics Subject Classification. Primary 54G05; Secondary 54H25. Key words and phrases. Extremally disconnected, retracts, fixed points. 
It is the goal of this paper to generalize this result to arbitrary maps on compact extremally disconnected spaces, and the following will be obtained:

Let $X$ be a compact extremally disconnected space and assume $\varphi: X \rightarrow X$ is a continuous map. Define

$$
P^{n}=\left\{x: \varphi^{n}(x)=x \text { and } m<n \rightarrow \varphi^{m}(x) \neq x\right\} .
$$

We usually denote $P^{1}$ by $F_{\varphi}$, the set of fixed points of the map $\varphi$. We will show a construction of a clopen subset $C \subset X$ with the following properties.

(1) $\varphi(C) \subset C$ and $\varphi(X-C) \subset X-C$.

(2) $F_{\varphi} \subset C$ and if $\varphi$ is injective then $F=C$.

(3) $F_{\varphi}$ is a retract of $C$, in fact there exists a retraction $r: C \rightarrow F_{\varphi}$ such that $r=r \circ \varphi$.

In particular, the subspace $F_{\varphi}$ is extremally disconnected.

(4) If $U$ is an open subset of $X$ with $\varphi(U) \subset U$, then: $U$ contains a fixed point if and only if $U \cap C \neq \varnothing$.

(5) $X-C$ is the union of three clopen sets $C_{i}$ with $\varphi\left(C_{i}\right) \cap C_{i}=\varnothing$, for all $i$.

The subsets $P^{n}$ (where $n$ is a prime number) have the following properties

(6) Each $P^{n}$ is compact and a retract of $X$. (Note that this implies that periodic points of order $n$ cannot accumulate to periodic points of different order. Another implication is that $P^{n}$ is extremally disconnected.)

(7) The set $\operatorname{cl} \bigcup\left\{P^{n}: n \geq 1\right.$ and $n$ is prime $\}$ is a retract of $X$. So, this subspace is also extremally disconnected.

These results indicate that the subspaces $P^{n}$ and the subspace $\operatorname{cl} \bigcup\left\{P^{n}: n \geq\right.$ 1) are fundamentally associated with the extremally disconnected structure of the underlying space. Recall that closed subspaces of extremally disconnected spaces need not be extremally disconnected.

In this paper machinery is built to investigate the structure of the continuous maps on these spaces. But this machinery not only works for compact extremally disconnected spaces, but can also be used for other classes of spaces, such as the class of basically disconnected spaces. Even the class of $F$-spaces can be put in this machinery, provided the class of maps is restricted. If this machinery is applied to the class of $F$-spaces, results appear which seem to be new. For example : it will be proved that under $\mathrm{CH}$, the fixed-points set of an open map on $\omega^{*}$ is a retract.

After the first version of this this manuscript was prepared, more results in this area were discovered and published. After some hesitation I have decided to add these results to this final version, as these results seem to fit so well in the machinery that is developed. Moreover, the cross references between these papers are not correct anymore.

I am indebted to Klaas Pieter Hart for the reading of the manuscript and for the many valuable suggestions.

\section{Preliminaries}

In this section we review background material on extremally disconnected spaces and mappings defined on them. A good deal of this material comes from 
[ $\left.K_{1}\right]$ and $[A, A, K]$. These papers were geared toward operator theory; we shall give a more topological form of these results.

Throughout this paper $X$ denotes a compact zero-dimensional space and maps and functions are assumed to be continuous, unless the contrary is stated explicitly. $B(X)$ denotes its Boolean algebra of clopen sets. Our main objects of concern are the extremally disconnected spaces and the basically disconnected spaces. We assume that the reader is familiar with these classes of spaces. We recall that each of the following properties may be used to define the extremally disconnected spaces.

- The inclusion order on $B(X)$ is complete.

- Closures of open sets are open.

- Disjoint open sets have disjoint closures.

- Dense subsets of $X$ are $C^{*}$-embedded.

- Open subsets of $X$ are $C^{*}$-embedded.

Note that the Čech-Stone compactification of an extremally disconnected space is extremally disconnected.

The class of basically disconnected spaces can be defined in the following way.

- The inclusion order on $B(X)$ is countably complete.

- Closures of cozerosets are open.

A space is called $\kappa$-basically disconnected if the inclusion order on $B(X)$ is $\kappa$-complete, i.e. if every $A \subset B(X)$ with $\operatorname{card}(A)<\kappa$ has a supremum. Note that the class of basically disconnected spaces coincides with the class of $\omega_{1}$-basically disconnected spaces. A space $X$ is extremally disconnected if the space is $\kappa$-basically disconnected for all $\kappa$.

Finally, a compact space $X$ is called an $F$-space, if one of the following equivalent properties is satisfied.

- Disjoint cozerosets have disjoint closures.

- Each cozeroset of $X$ is $C^{*}$-embedded.

- Each $F_{\sigma}$-subset of $X$ is $C^{*}$-embedded.

Evidently, we have the following implications between these types of spaces: An extremally disconnected space is $\kappa$-basically disconnected, a $\kappa$-basically disconnected space is basically disconnected, and a basically disconnected space is $F$.

Recall that a subset $A \subset X$ is called a $P$-set if the neighborhood system of $A$ is closed under countable intersections. In general, $A \subset X$ is called a $P_{\kappa}$-set, if the neighborhood system is closed under fewer than $\kappa$ intersections. In particular, a $P$-set is a $P_{\omega_{1}}$-set.

Subspaces of extremally disconnected spaces need not be extremally disconnected anymore, as easy examples show. We collect some well-known facts about properties of subspaces.

Lemma 1.1. (1) A closed subspace of a compact F-space is an F-space. Closed subspaces of extremally disconnected spaces (basically disconnected spaces) need not be extremally disconnected ( basically disconnected ), but are F-spaces. 
(2) If $X$ is a compact $\kappa$-basically disconnected space and $A \subset X$ is a $P_{\kappa}$-set, then $A$ is compact $\kappa$-basically disconnected. In particular, $P$-sets in basically disconnected spaces are basically disconnected.

(3) If $A$ is a retract of the compact extremally disconnected space $X$, then $A$ is extremally disconnected.

(4) If $A$ is a retract of the compact $\kappa$-basically disconnected space $X$, then $A$ is $\kappa$-basically disconnected.

We collect the following results on partitions. Let $X$ be a zerodimensional space with a map $\varphi$. A subset $A$ of $X$ is said to have a $(k)$-partition in $X$, (or, can be $k$-partioned in $X$ with respect to $\varphi$ ) if there exists a family $\left\{C_{1}, \ldots, C_{k}\right\}$ with the following properties:

(1) Each $C_{i}$ is (relatively) clopen in $A$, and $A=\bigcup C_{i}$.

(2) $C_{i} \cap C_{j}=\varnothing$, if $i \neq j$.

(3) $\varphi\left(C_{i}\right) \cap C_{i}$, for all $i$.

Proposition 1.2. (1) [K,S] Let $X$ be a compact zerodimensional space and $\varphi$ a map without fixed points. Then $X$ has a 3-partition with respect to $\varphi$.

(2) [K,S] Let $X$ be a zerodimensional $\sigma$-compact space and $\varphi: X \rightarrow X a$ continuous map. Then $\varphi$ has fixed points iff $\beta \varphi$ has fixed points.

(3) $[\mathrm{A}, \mathrm{A}, \mathrm{K}]$ Let $A$ be a compact subspace of an extremally disconnected space $X$, and let $\varphi$ be a map of $X$ such that $A$ contains no fixed points of $F_{\varphi}$. Then $A$ has a 3-partition

(4) $\left[\mathrm{K}_{2}\right]$ Let $X$ be a compact zerodimensional space and $\varphi$ an injective map without fixed points. Let $A$ and $B$ be closed subsets of $X$ with $A \subset B$.

Then every 3-partition $A_{1}, A_{2}, A_{3}$ of pairwise disjoint (relatively) clopen subsets of $A$ can be extended to a 3-partition $B_{1}, B_{2}, B_{3}$ of (relatively) clopen subsets of $B$ with $B_{i} \cap A=A_{i}$, for all $i$.

\section{THE SHARP OPERATOR}

For the moment a compact zerodimensional space $X$ with selfmap $\varphi$ is fixed. A subset $A \subset X$ is called $\varphi$-invariant, (resp. $\varphi^{-1}$-invariant) if $\varphi(A) \subset A$, (resp. $\left.\varphi^{-1}(A) \subset A\right)$.

For a given subset $A \subset X$, there exists a subset $B$ with the following properties:

- $A \subset B, B$ is closed and $\varphi^{-1}(B) \subset B$.

- If $A \subset D, D$ is closed and $\varphi^{-1}(D) \subset D$, then $B \subset D$.

This particular subset $B$ is called $A^{\#}$, and clearly it is the smallest closed $\varphi^{-1}$ invariant subset of $X$ containing $A$. The set $A^{\#}$ can be constructed in the following way.

Define by transfinite induction sets $A_{\alpha}$ :

(1) $A_{0}=\operatorname{cl} A$.

(2) $A_{\alpha+1}=A_{\alpha} \cup \varphi^{-1}\left(A_{\alpha}\right)$.

(3) For limit ordinals $\beta$, put $A_{\beta}=\operatorname{cl}\left(\bigcup\left\{A_{\alpha}: \alpha<\beta\right\}\right)$.

In this way an increasing sequence $\left\{A_{\alpha}: \alpha \in\right.$ Ord $\}$ of closed subsets appears. If $A_{\alpha+1}=A_{\alpha}$ then obviously $A^{\#}=A_{\alpha}$. Note that the set $A^{\#}$ depends on the $\operatorname{map} \varphi$ (what is not discernible in the notation). In the following lemma some of the basic properties of the \# -operator are collected, all of which are easy to verify. 
Lemma $2.1([\mathrm{~A}, \mathrm{~A}, \mathrm{~K}])$. (1) $A \subset B \subset A^{\#} \Rightarrow A^{\#}=B^{\#}$.

(2) $(A \cup B)^{\#}=A^{\#} \cup B^{\#}$.

(3) $x \in A_{\alpha+1} \Rightarrow \varphi(x) \in A_{\alpha}$.

(4) If $\beta$ is a limit, then:

$x \in A_{\beta}-\bigcup\left\{A_{\alpha}: \alpha<\beta\right\} \Rightarrow \varphi(x) \in A_{\beta}-\bigcup\left\{A_{\alpha}: \alpha<\beta\right\}$.

(5) $\varphi\left(A^{\#}\right) \subset A^{\#}$ if and only if $\varphi(A) \subset A^{\#}$

(6) $A^{\#} \cap B^{\#}=\varnothing \Rightarrow \varphi\left(A^{\#}\right) \cap B=\varnothing=\varphi\left(B^{\#}\right) \cap A$.

(7) $\varphi\left(X-A^{\#}\right) \subset X-A^{\#}$.

Lemma $2.2([\mathrm{~A}, \mathrm{~A}, \mathrm{~K}])$. If $\varphi$ is an open map, then $A^{\#}=A_{\omega_{0}}$.

Proof. It suffices to show that the set $A_{\omega_{0}}$ is $\varphi^{-1}$-invariant. Assume $\varphi(x) \in$ $A_{\omega_{0}}$, then for every open neighborhood $U_{x}$ of $x$ (as $\varphi\left(U_{x}\right)$ is open):

$\varphi\left(U_{x}\right) \cap \bigcup\left\{A_{n}: n \in \omega_{0}\right\} \neq \varnothing$. If $\varphi\left(U_{x}\right) \cap A_{n} \neq \varnothing$ then $U_{x} \cap A_{n+1} \neq \varnothing$.

We conclude: $U_{x} \cap \bigcup\left\{A_{n}: n \in \omega_{0}\right\} \neq \varnothing$ for all open $U_{x}$, i.e. $x \in A_{\omega_{0}}$.

As we are working on the class of zerodimensional spaces we are particularly interested in selfmaps $\varphi$ on spaces $X$ with the property:

$C$ clopen in $X$ implies $C^{\#}$ clopen in $X$.

In $\left[\mathrm{V}_{2}\right]$ the following class of maps was introduced:

Let $\kappa$ be a cardinal (i.e. an initial ordinal). A map $\varphi$ on the space $X$ is called a \# $\#$-map, if in the transfinite definition of the set $A^{\#}$, the identity $A_{\alpha+1}=A_{\alpha}$ in the sequence $\left\{A_{\alpha}: \alpha \in\right.$ Ord $\}$ appears on a level $\gamma$ with $\gamma<\kappa$, for all $A \subset X$. Note that Lemma 2.2 implies that open maps are $\# \omega_{1}$.

The following lemma is easy to verify.

Lemma 2.3. Let $\varphi$ be a map on the compact space $X$. In each of the following cases:

- $X$ is extremally disconnected.

- $X$ is $\kappa$-basically disconnected and $\varphi$ is a \# $\kappa$-map.

- $X$ is basically disconnected and $\varphi$ is an open map.

The property " $C$ clopen in $X$ implies $C$ " clopen in $X$ " is valid.

\section{THE FIXED-POINT SET}

Fix a space $X$ and a selfmap $\varphi: X \rightarrow X$. The set $F_{\varphi}=\{x: \varphi(x)=x\}$ is the fixed-point set of $\varphi$. The main result of this section is that in many cases the set $F_{\varphi}$ is a retract of the set $F_{\varphi}{ }^{\#}$. To obtain this result we need the following lemma.

Lemma 3.1. Let $\varphi$ be a selfmap of the compact space $X$. The property

$$
A \cap F_{\varphi}=\varnothing \Rightarrow A^{\#} \cap F_{\varphi}=\varnothing, \text { for all closed sets } A \subset X
$$

is valid in any one of the following cases:

(1) $X$ is an $F$-space and $\varphi$ is open.

(2) $X$ is $\kappa$-basically disconnected and $\varphi$ is a \# $\kappa$-map.

(3) $X$ is extremally disconnected. 
Proof. Let $A \subset X$ be a closed set with $A \cap F_{\varphi}=\varnothing . A$ can be covered with finitely many clopen sets $C_{i}$, with the property: $\varphi\left(C_{i}\right) \cap C_{i}=\varnothing$. Since $A^{\#} \subset$ $\cup C_{i}^{\#}$ it suffices to show

$$
C \cap F_{\varphi}=\varnothing \text { implies } C^{\#} \cap F_{\varphi}=\varnothing
$$

for clopen sets $C$ with the additional property $\varphi(C) \cap C=\varnothing$.

In each of the three cases a continuous function $f: C^{\#} \rightarrow\{0,1,2\}$ is constructed with: $f(\varphi(x)) \neq f(x)$, for all $x$ with $\{x, \varphi(x)\} \subset C^{\#}$. (Of course this implies that $C^{\#} \cap F_{\varphi}=\varnothing$.) Let $C$ be clopen with $\varphi(C) \cap C=\varnothing$. Put $D_{0}=C$ and $D_{n+1}=\varphi^{-1}\left(C_{n}\right)-C_{n}$

Then $\bigcup\left\{D_{n}: n \in \omega_{0}\right\}=\bigcup\left\{C_{n}: \in \omega_{0}\right\}$. The map $f$ is defined by $f(x)=0$ if $x \in C=D_{0}, f(x)=1$ if $x \in D_{2 k}$ and $f(x)=2$ if $x \in D_{2 k+1}$. Note that $f$ is continuous on $\bigcup C_{n}$ and has the required property. In each of the cases we proceed as follows.

(1) Consider the case that $X$ is an $F$-space. The set $\cup C_{n}$ is $C^{*}$-embedded in $X$, so $f$ has a continuous extension to $\operatorname{cl}\left(\cup C_{n}\right)=C^{\#}$, as the map $\varphi$ is open. Note that

$$
O_{1}=\bigcup\left\{D_{2 k}: k \geq 1\right\} \text { and } O_{2}=\bigcup\left\{D_{2 k+1}: k \leq 0\right\}
$$

are two disjoint (relatively) clopen sets of $\cup C_{n}$, so they have disjoint closures in $C^{\#}$. It follows easily that the extension of the map $f$ to $C^{\#}$ only has the values 1,2 on $\operatorname{cl}\left(\cup C_{n}\right)-\cup C_{n}$, and satisfies the required inequality.

(2) If the space $X$ is $\kappa$-basically disconnected, then the space is $F$ and again the map $f$ can be extended to $\operatorname{cl}\left(\cup C_{n}\right)$. On the remainder $\operatorname{cl}\left(\bigcup C_{n}\right)-\bigcup C_{n}$ the function $f$ only admits the values 1 and 2 . Put $D_{\alpha+1}=\varphi^{-1}\left(C_{\alpha}\right)-C_{\alpha}$, and extend $f$ to $\bigcup D_{\alpha}$ by

$$
f(x)=i \leftrightarrow f(\varphi(x))=3-i, \quad i \in\{1,2\} .
$$

Since the space is $\kappa$-basically disconnected the particular map $f$ can be extended to limit-levels $\gamma<\kappa$. As the map is $\# \kappa$, it can be extended to $C^{\#}$.

(3) This is proved in $[A, A, K]$, but it also follows from the previous case.

The notion of an attractor is well-known. However, I would like to distinguish the following types of attractors. A point $x \in F_{\varphi}$ is called an

- attractor, if for every neighborhood $V_{x}$ of $x$ there exists a open neighborhood $U_{x}$ with: $\varphi\left(U_{x}\right) \subset U_{x} \subset V_{x}$.

- strong attractor, if for every neighborhood $V_{x}$ of $x$ there exists a clopen neighborhood $U_{x}$ with: $\varphi\left(U_{x}\right) \subset U_{x} \subset V_{x}$.

- weak attractor, if for every neighborhood $V_{x}$ of $x$ there exists a neighborhood $U_{x}$ with: $\varphi\left(U_{x}\right) \subset U_{x} \subset V_{x}$.

I do not have examples that witness the difference between these notions. The following result suggests that it might be possible that in the class of $F$ spaces one might find an example of a space and a map with a fixed point that is an attractor but not a strong attractor. It was proved by Frolik that the fixed points of maps of compact extremally disconnected spaces always are strong attractors. 
Proposition 3.2. (1) Each fixed point of an open map $\varphi$ on a $F$-space $X$ is an attractor.

(2) Each fixed point of a \#к-map $\varphi$ on a $\kappa$-basically disconnected space $X$ is a strong attractor.

(3) Each fixed point of a map $\varphi$ on a extremally disconnected space $X$ is a strong attractor $[\mathrm{F}]$.

Proof. Assume $\varphi(x)=x$, and let $U$ be a clopen neighborhood of $X$. The set

$$
A=\{t \in U: \varphi(t) \notin U\}=U \cap \varphi^{-1}(X-U)
$$

is clopen and disjoint from $F_{\varphi}$. We conclude in each of the cases that $A^{\#} \cap F_{\varphi}=$ $\varnothing$. It is easy to verify that: $x \in U-A^{\#}$ and $\varphi\left(U-A^{\#}\right) \subset U-A^{\#}$.

Note that in the second and third case the set $U-A^{\#}$ is clopen, in the first case one only can conclude that the set $U-A^{\#}$ is closed. The conclusion follows.

Theorem 3.3. In each of the following three cases:

(1) $X$ is an $F$-space and $\varphi$ is open.

(2) $X$ is $\kappa$-basically disconnected and $\varphi$ is a \# $\kappa$-map.

(3) $X$ is extremally disconnected.

The set $F_{\varphi}$ is a retract of $F_{\varphi}$. There even exists a retraction $r: F_{\varphi}^{\#} \rightarrow F_{\varphi}$ with the property : $r=r \circ \varphi$.

Proof. It will be checked that the map $A \rightarrow A^{\#}$ is a Boolean map from the clopen sets of $F_{\varphi}$ to the clopen sets of $F_{\varphi}{ }^{\#}$ with the property: $A^{\#} \cap F_{\varphi}=A$. The dual map is the required retraction. We have to check:

$$
A \text { (relatively) clopen in } F_{\varphi} \Rightarrow A^{\#} \text { (relatively) clopen in } F_{\varphi}^{\#} \text {. }
$$

For this it is enough to check that

$$
A \text { (relatively) clopen in } F_{\varphi} \Rightarrow A^{\#} \cap\left(F_{\varphi}-A\right)^{\#}=\varnothing .
$$

Once this is checked, it follows immediatly that the map $A \rightarrow A^{\#}$ is Boolean. (After all, the sharp operator commutes with the union.) Let $A$ be a (relatively) clopen set of $F_{\varphi}$, and put $B=F_{\varphi}-A$. In each of the cases we proceed as follows.

(1) It is clear that

$$
\bigcup\left\{\varphi^{-n}(A): n \in \omega_{0}\right\} \cap \bigcup\left\{\varphi^{-n}(B): n \in \omega_{0}\right\}=\varnothing .
$$

The sets $\bigcup\left\{\varphi^{-n}(A): n \in \omega_{0}\right\}$ and $\bigcup\left\{\varphi^{-n}(B): n \in \omega_{0}\right\}$ are disjoint $F_{\sigma}$ sets in $X$. Since $A^{\#}=\operatorname{cl}\left(\bigcup\left\{\varphi^{-n}(A): n \in \omega_{0}\right\}\right)$, it suffices to show that the sets $\bigcup\left\{\varphi^{-n}(A): n \in \omega_{0}\right\}$ and $\bigcup\left\{\varphi^{-n}(B): n \in \omega_{0}\right\}$ are separated in $X$. By Proposition 3.2 there exists open sets $U$ and $V$, with:

- $A \subset U, B \subset V, U \cap V=\varnothing$.

- $\varphi(U) \subset U$ and $\varphi(V) \subset V$.

It is the second property that implies that for all $u \in U$, and for all $k: \varphi^{k}(u) \notin$ $V$. Likewise for the set $V$. Therefore

$$
\bigcup\left\{\varphi^{-n}(U): n \in \omega_{0}\right\} \cap \bigcup\left\{\varphi^{-n}(V): n \in \omega_{0}\right\}=\varnothing .
$$


We conclude that the sets $\bigcup\left\{\varphi^{-n}(A): n \in \omega_{0}\right\}$ and $\bigcup\left\{\varphi^{-n}(B): n \in \omega_{0}\right\}$ are separated.

(2) In this case the sets $U$ and $V$ can be taken to be clopen, and therefore:

- The sets $\operatorname{cl}\left(\bigcup\left\{\varphi^{-n}(U): n \in \omega_{0}\right\}\right)$ and $\operatorname{cl}\left(\bigcup\left\{\varphi^{-n}(V): n \in \omega_{0}\right\}\right)$ are disjoint and clopen.

- $\varphi\left(\operatorname{cl}\left(\bigcup\left\{\varphi^{-n}(U): n \in \omega_{0}\right\}\right)\right) \subset \operatorname{cl}\left(\bigcup\left\{\varphi^{-n}(U): n \in \omega_{0}\right\}\right)$.

- $\varphi\left(\operatorname{cl}\left(\bigcup\left\{\varphi^{-n}(V): n \in \omega_{0}\right\}\right)\right) \subset \operatorname{cl}\left(\bigcup\left\{\varphi^{-n}(V): n \in \omega_{0}\right\}\right)$.

So one can continue the induction to any ordinal level $\gamma$ with $\gamma<\kappa$, and therefore

$$
A^{\#} \subset U^{\#}, B^{\#} \subset V^{\#} \text { and } U^{\#} \cap V^{\#}=\varnothing .
$$

The conclusion follows.

(3) This follows from the previous case.

The property $r=r \circ \varphi$ defines the retraction completely. Indeed, this formula determines the retraction $r$ as a map $r: \cup \varphi^{-n}\left(F_{\varphi}\right) \rightarrow F_{\varphi}$. The (weak) attractor property of the fixed points implies that this particular map is continuous in the points of $F_{\varphi}$. If the space is an $F$-space, this retraction can be extended to $\operatorname{cl}\left(\bigcup \varphi^{-n}\left(F_{\varphi}\right)\right)$. It is remarkable that, in the case that $X$ is $\kappa$ basically disconnected, the map $r$ can be extended to higher limit levels. After all, $F_{\gamma}$ sets with $\gamma<\kappa$ need not be $C^{*}$-embedded in a $\kappa$-basically disconnected space. But this is the place where it is used that the fixed points are strong attractors. A union of less than $\kappa$ many clopen sets is $C^{*}$-embedded in such a space.

\section{THE SET $F_{\varphi}{ }^{\#}$ FOR $F$-SPACES.}

The goal of this section is to show that the set $F_{\varphi}{ }^{\#}$ has the property that it is remote from its complement, and this in several senses. For example, it was proved in $[\mathrm{A}, \mathrm{A}, \mathrm{K}]$ that the set $F_{\varphi}{ }^{\#}$ is clopen, in the case that $X$ is compact extremally disconnected, i.e. as remote from its complement as possible. In $\left[\mathrm{V}_{2}\right]$ this result was generalized in the following way: if the space $X$ is compact $\kappa$-basically disconnected and the map $\varphi$ is $\# \kappa$ then $F_{\varphi}{ }^{\#}$ is a $P_{\kappa}$-set.

We will show similar results in the case that $X$ is a $F$-space. We begin with some lemmas that also present remoteness-type properties of the set $F_{\varphi}^{\#}$.

Lemma 4.1. Let $X$ be a compact space and $\varphi$ a selfmap of $X$.

(1) Assume $U \subset X$ is an open subset of $X$ with the properties

$$
\varphi(U) \subset U \text { and } U \cap F_{\varphi}=\varnothing \text {. }
$$

Then $U \cap F_{\varphi}{ }^{\#}=\varnothing$.

(2) Assume $C \subset X$ is a clopen subset of $X$ with the properties

$$
\varphi(C) \subset C \text { and } C \cap F_{\varphi}=\varnothing \text {. }
$$

If either $X$ is compact extremally disconnected or $X$ is compact $\kappa$ basically disconnected and the map $\varphi$ is \# $\kappa$ then $C^{\#} \cap F_{\varphi}^{\#}=\varnothing$.

Proof. Assume $U \cap F_{\varphi}^{\#} \neq \varnothing$. Put $\alpha_{0}=\min \left\{\alpha: U \cap\left(F_{\varphi}\right)_{\alpha} \neq \varnothing\right\}$. The property $\varphi(U) \subset U$ implies that $\alpha_{0}$ is not a successor ordinal and $U$ open implies that $\alpha_{0}$ is not a limit ordinal. So $\alpha_{0}=0$, i.e. $U \cap F_{\varphi} \neq \varnothing$. To prove 
the second statement, observe that the proof of Lemma 3.1 implies that there exists a continuous function $f: C^{\#} \rightarrow\{0,1,2\}$ such that $f(x) \neq f(\varphi(x))$ if $\{x, \varphi(x)\} \subset C^{\#}$. This implies that $C^{\#} \cap F=\varnothing$. The property $\varphi(C) \subset C$ implies $\varphi\left(C^{\#}\right) \subset C^{\#}$ and we can conclude from the first part of this lemma that $C^{\#} \cap F_{\varphi}^{\#}=\varnothing$.

Next we collect some properties of the set $F_{\varphi}{ }^{\#}$ in the class of compact zerodimensional $F$-spaces.

Theorem 4.2. Let $X$ be a compact $F$-space and $\varphi$ a continuous map.

(1) $[\mathrm{H}, \mathrm{V}]$ If $\varphi$ is injective then $F_{\varphi}{ }^{\#}=F_{\varphi}$ and $F_{\varphi}$ is a $P$-set of $X$.

(2) If the map $\varphi$ is open, then $F_{\varphi}^{\#}$ is a P-set of $X$.

Proof. We only verify the second statement. First observe the following: If $A$ is a $\sigma$-compact subset of $X$ with $A \cap{F_{\varphi}}^{\#}=\varnothing$ then there exists a cozeroset $U$ with $A \subset U \subset X-F_{\varphi}{ }^{\#}$ and $\varphi(U) \subset U$.

To see this, note first that every $\sigma$-compact subset $A \subset X-F_{\varphi}{ }^{\#}$ is contained in a cozeroset $U$ with $A \subset U \subset X-F_{\varphi}{ }^{\#}$. Choose by induction cozerosets $U^{n}$ such that

- $A \subset U^{0} \subset X-F_{\varphi}{ }^{\#}$.

- $U^{n} \cup \varphi\left(U^{n}\right) \subset U^{n+1} \subset X-F_{\varphi}^{\#}$.

If we put $U=\bigcup U^{n}$, then this is the required cozeroset. Therefore, if we can check the following:

"Every cozeroset $U \subset X$ with $\varphi(U) \subset U$ and $U \subset X-F_{\varphi}$ " has the property:

$$
\operatorname{cl} U \cap F_{\varphi}^{\#}=\varnothing "
$$

then it would follow that $F_{\varphi}^{\#}$ is a $P$-set.

Consider the cozeroset $U$. This set is $\sigma$-compact, so $C^{*}$-embedded in $X$. It follows from Proposition 1.2 that the map $\beta(\varphi \mid U)$ has no fixed points, in particular,

$$
\operatorname{cl} U \cap F=\varnothing .
$$

Note also that

$$
\varphi(\operatorname{cl} U) \subset \operatorname{cl} U .
$$

(Note that this already proves the first claim, i.e. the case with $\varphi$ injective) The fixed points of the map $\varphi$ are attractors, so there exist an open set $O$ with $F_{\varphi} \subset O \subset X-\operatorname{cl} U$ and $\varphi(O) \subset O$. But then

$$
U \cap \bigcup\left\{\varphi^{-k}(O): k \in \omega_{0}\right\}=\varnothing
$$

It can be concluded that the disjoint $F_{\sigma}$-sets $U$ and $U\left\{\varphi^{-k}\left(F_{\varphi}\right): k \in \omega_{0}\right\}$ are separated, and therefore have disjoint closures, i.e. $\operatorname{cl} U \cap F_{\varphi}{ }^{\#}=\varnothing$. The conclusion follows.

The conclusion is that there is some tendency within the class of $F$-spaces, for the fixed-points sets to become $P$-sets. And this tendency becomes stronger by higher degrees of disconnectedness of the underlying space. The previous 
theorem can be used to present new methods to find points of different topological type within an $F$-space $X$. The argument is similar to the argument of Frolik.

Theorem 4.3. Let $X$ be a compact zerodimensional $F$-space. Let $C_{n}$ be a clopen subset of $X$ and assume that the subspace

$$
D=\operatorname{cl}\left[\bigcup\left\{C_{n}: n \in \omega_{0}\right\}\right]-\bigcup\left\{C_{n}: n \in \omega_{0}\right\}
$$

contains a copy of $X$. Then $X$ is not homogenuous.

Proof. Let $g: X \rightarrow X$ be an embedding with $g(X) \subset D$. Let $x_{0}$ be a point of $X$.

Claim: There does not exists an embedding $h: X \rightarrow X$ with $h\left(g\left(x_{0}\right)\right)=x_{0}$ Indeed, if such a map would exist, then the map $k=g \circ h$ is injective and $x_{0}$ is a fixed point of the map $k$. It follows that $D$ would contain the non-empty $P$-set $F_{k}$. This is clearly impossible. Contradiction.

We collect some results of properties of selfmaps of the most elementary $F$-space that exists, the space $\beta \omega-\omega=\omega^{*}$. Recall:

Lemma $4.4[\mathrm{vM}]$. Under the continuum hypothesis $[\mathrm{CH}]$ all $P$-sets of $\omega^{*}$ are retracts of $\omega^{*}$.

Proposition 4.5. Let $\varphi$ be a continuous selfmap on $\omega^{*}$. If $[\mathrm{CH}]$ and either the map $\varphi$ is open or injective then $F_{\varphi}$ is a retract of $\omega^{*}$.

Proof. If the map is injective it follows from the first statement in Theorem 4.2 and the previous lemma. If the map is open it follows from the following lemma and the second statement in Theorem 4.2

Theorem 4.6. Let $\varphi$ be an embedding of $\omega^{*}$ into itself. If $[\mathrm{CH}]$ then $\omega^{*}-F_{\varphi}$ is the union of three disjoint open sets $U_{i}, i=0,1,2$, with the property $U_{i} \cap$ $\varphi\left(U_{i}\right)=\varnothing$, for all $i$.

Proof. The continuum hypothesis implies that the complement of the $P$-set $F_{\varphi}$ can be written as an increasing union of $\omega_{1}$ clopen sets. Say $\omega^{*}-F_{\varphi}=\bigcup\left\{C_{\alpha}\right.$ : $\left.\alpha<\omega_{1}\right\}$, with $C_{\alpha} \subset C_{\beta}$ for $\alpha<\beta$.

Replace each $C_{\alpha}$ by the closure of its $\varphi$-saturation:

$$
D_{\alpha}=\mathrm{cl}\left[\bigcup\left\{\varphi^{n}\left(C_{\alpha}\right): n \in \omega_{0}\right\}\right] .
$$

Then $\omega^{*}-F_{\varphi}$ is written as an increasing union of closed sets $D_{\alpha}$, with the additional property: $\varphi\left(D_{\alpha}\right) \subset D_{\alpha}$. Moreover, we can enlarge the sets $D_{\alpha}$ even more such that:

$$
D_{\alpha} \subset \text { int } D_{\beta}, \quad \text { for } \alpha<\beta \text {. }
$$

By induction the set $D_{\alpha}$ is covered by sets $\left\{U_{\alpha}^{i}: i=0,1,2\right\}$ which are clopen in $\omega^{*}$, in the following way:

Find a 3-partition $\left\{D_{0}^{i}: i=0,1,2\right\}$ of $D_{0}$, and choose disjoint clopen (in $\left.\omega^{*}\right)$ sets $\left\{U_{0}^{i}: i=0,1,2\right\}$ such that:

$$
U_{0}^{i} \cap D_{0}=D_{0}^{i}, \quad U_{0}^{i} \subset \operatorname{int}\left(D_{1}\right) \text { and } \varphi\left(U_{0}^{i}\right) \cap U_{0}^{i}=\varnothing .
$$


According to Proposition 1.2 it is possible to extend the partition $\left\{U_{0}^{i}: i=\right.$ $0,1,2\}$ to a partition $\left\{D_{1}^{i}: i=0,1,2\right\}$ of $D_{1}$ and next this partition can be enlarged to pairwise disjoint clopen sets $\left\{U_{1}^{i}: i=0,1,2\right\}$.

The construction on the rest of the non-limit levels is identical to the one above.

On a limit level $\beta$ we proceed as follows. The construction implies that the set

$$
D^{\beta}=\bigcup\left\{D_{\alpha}: \alpha<\beta\right\}\left(\subset D_{\beta}\right)
$$

has the property

$$
D^{\beta}=\bigcup\left\{\bigcup\left\{U_{\alpha}^{i}: i=0,1,2\right\}: \alpha<\beta\right\} .
$$

Note that $D^{\beta}$ is an open $F_{\sigma}$ set, $\varphi\left(D^{\beta}\right) \subset D^{\beta}$, and that $D^{\beta}$ is $C^{*}$-embedded in $\omega^{*}$. $D^{\beta}$ has a natural partition, namely: $\left\{\bigcup\left\{U_{\alpha}^{i}: \alpha<\beta\right\}: i=0,1,2\right\}$ of sets which are open in $\omega^{*}$.

It follows that $\left\{\operatorname{cl}\left[\bigcup\left\{U_{\alpha}^{i}: \alpha<\beta\right\}\right]: i=0,1,2\right\}$ is a partition of $\operatorname{cl} D^{\beta}$. As above extend this to a partition $\left\{U_{\beta}^{i}: i=0,1,2\right\}$ of $D_{\beta}$ into sets clopen in $\omega^{*}$. This ends the induction. If we put $U^{i}=\bigcup\left\{U_{\alpha}^{i}: \alpha<\omega_{1}\right\}$, then the required partition of $\omega^{*}-F_{\varphi}$ is defined.

Question 4.7. (1) Let $X$ be an $F$-space. Is $F_{\varphi}{ }^{\#}$ a P-set, for arbitrary maps $\varphi$ ?

(2) Is $[\mathrm{CH}]$ essential the previous theorem?

(3) Is it consistent with $[\mathrm{CH}]$, that all fixed-point sets of $\omega^{*}$ are retracts? And what can be said in ZFC?

To motivate these questions, in the next sections most of these questions will be answered for the more restricted classes of basically or extremally disconnected spaces.

\section{THE SET $F_{\varphi} \#$ FOR BASICALly DISCONNECTED SPACES.}

In this section we discuss the class of compact $\kappa$-basically disconnected spaces. Several results in this section already appeared in $\left[\mathrm{V}_{2}\right]$. However, in this paper, references to an earlier version of this paper appeared which are no longer valid. To make at least one of the papers selfcontained, the proofs will be repeated in this paper. We also add some new results.

Theorem 5.1. Let $X$ be a compact $\kappa$-basically disconnected space and let $\varphi$ be a selfmap of $X$. Then:

(1) $F_{\varphi}^{*}$ is a P-set.

(2) If $\varphi$ is a \# $\kappa$-map then $F_{\varphi}{ }^{\#}$ is a $P_{\kappa}$-set.

Proof. (1) Let $A$ be a closed $F_{\sigma}$-set disjoint from $F_{\varphi}^{\#}$. In Theorem 4.2 we saw that there exists a cozeroset $U$ with $\varphi(U) \subset U$ and $U \cap F_{\varphi}^{\#}=\varnothing$. As in Theorem 4.2 we can conclude that $\operatorname{cl} U \cap F_{\varphi}=\varnothing$. But in this case we even have that $\operatorname{cl} U$ is clopen, and we can use the first statement in Lemma 4.1 to conclude that $\operatorname{cl} U \cap F_{\varphi}{ }^{\#}=\varnothing$. We see that $\operatorname{cl} A \cap F_{\varphi}^{\#}=\varnothing$. It follows that the set $F_{\varphi}^{\#}$ is a $P$-set. 
(2) Assume $\left\{A_{\alpha}: \alpha<\gamma\right\}$ is a family of closed sets with $A_{\alpha} \cap F_{\varphi}{ }^{\#}=\varnothing$, for all $\alpha<\gamma$ were $\gamma<\kappa$.

By induction we construct clopen sets $C_{\alpha}$ such that:

- $A_{\alpha} \subset C_{\alpha} \subset X-F_{\varphi}^{\#}$.

- $C_{\alpha} \subset C_{\beta}$, for $\alpha<\beta<\gamma$.

- $C_{\alpha}$ is both $\varphi$-invariant and $\varphi^{-1}$-invariant.

Together with the clopen sets a partition $\left\{D_{\alpha}^{0}, D_{\alpha}^{1}, D_{\alpha}^{2}\right\}$ of $C_{\alpha}$ is constructed which satisfies the following properties.

- $D_{\alpha}^{0} \cup D_{\alpha}^{1} \cup D_{\alpha}^{2}=C_{\alpha}$.

- $D_{\alpha}^{i} \cap D_{\alpha}^{j}=\varnothing,(i \neq j)$.

- $\varphi\left(D_{\alpha}^{i}\right) \cap D_{\alpha}^{i}=\varnothing$.

- $D_{\beta}^{i} \cap C_{\alpha}=D_{\alpha}^{i}$, for $\alpha<\beta<\gamma$.

To construct these clopen sets we proceed as follows In (1) of the theorem a clopen set $C$ is constructed, with $A_{0} \subset C \subset X-F_{\varphi}{ }^{\#}$ and $\varphi(C) \subset C$. It follows from the second statement in Lemma 4.1 that $C^{\#} \cap F_{\varphi}{ }^{\#}=\varnothing$. Note that $\varphi(C) \subset C$ implies that $C^{\#}$ is $\varphi$-invariant. Put $C_{0}=C^{\#}$ and fix a 3-partition $\left\{D_{0}^{0}, D_{0}^{1}, D_{0}^{2}\right\}$ of. $C_{0}$. Assume for $\alpha<\beta$ the sets $C_{\alpha}$ and the partitions are defined, where $\beta<\kappa$.

First consider the set $U=\bigcup\left\{C_{\alpha}: \alpha<\beta\right\}$. This set is a union of less than $\kappa$ clopen sets, therefore $U$ is $C^{*}$-embedded and has a clopen closure. Note that $\varphi(U) \subset U$ and also $\operatorname{cl} U \cap F_{\varphi}=\varnothing$. Indeed, if we define $U_{i}=\bigcup\left\{D_{\alpha}^{i}: \alpha<\beta\right.$, $(i=0,1,2)$, then $U=U_{0} \cup U_{1} \cup U_{2}, U_{i} \cap U_{j}=\varnothing \quad(i \neq j)$ and $\varphi\left(U_{i}\right) \cap U_{i}=$ $\varnothing$.

It follows that $\beta(\varphi \mid U)$ has no fixed points and, as $U$ is $C^{*}$-embedded, we conclude that $\operatorname{cl} U \cap F_{\varphi}=\varnothing$. By Lemma 4.1 we conclude

$$
\operatorname{cl} U \cap F_{\varphi}^{\#}=\varnothing \text {. }
$$

The set $\operatorname{cl} U$ has a natural 3-partition, namely : $\left\{\operatorname{cl} U_{0}, \operatorname{cl} U_{1}, \operatorname{cl} U_{2}\right\}$. This corresponds to a continuous map $f: \operatorname{cl} U \rightarrow\{0,1,2\}$ with $f(x) \neq f(\varphi(x))$, where $f$ is defined by: $f\left(\operatorname{cl} U_{i}\right)=i$.

Note that by Lemma 4.1 we even have: $(\operatorname{cl} U)^{\#} \cap F_{\varphi}^{\#}=\varnothing$ and as in Lemma 3.1 the map $f$ can be extended to a map $f:(\operatorname{cl} U)^{\#} \rightarrow\{0,1,2\}$. This means that the 3 partition $\left\{\operatorname{cl} U_{0}, \operatorname{cl} U_{1}, \operatorname{cl} U_{2}\right\}$ of $\operatorname{cl} U$ can be enlarged to a 3-partition $E_{0}, E_{1}, E_{2}$ of the clopen set $(\operatorname{cl} U)^{\#}$.

Put $E=(\operatorname{cl} U)^{\#}$. Note that the clopen set $E=(\operatorname{cl} U)^{\#}$ is both $\varphi$ - and $\varphi^{-1}$-invariant. Consider the clopen subset $A_{\beta}-E$ of the compact $\kappa$-basically disconnected space $X-E$. As in the basic stap of the inductive proof, there exists a $\varphi$ and $\varphi^{-1}$-invariant clopen set $C_{0}$ with $A_{\beta}-E \subset C_{0} \subset X-E-F_{\varphi}^{\#}$ together with a 3 -partition $\left\{D_{0}^{0}, D_{0}^{1}, D_{0}^{2}\right\}$ of $C_{0}$. Finally, put

$$
C_{\beta}=(\operatorname{cl} U)^{\#} \cup C_{0}
$$

and $D_{\beta}^{i}=D_{i} \cup E_{i}$, for $i \in\{0,1,2\}$. This ends the inductive proof.

Corollary 5.2. Let $X$ be a compact $\kappa$-basically disconnected space and let $\varphi$ be a continuous selfmap of $X$. Then: 
(1) The space $F_{\varphi}{ }^{\#}$ is basically disconnected.

If, moreover, the map $\varphi$ is \# $\kappa$, then:

(2) The spaces $F_{\varphi}^{\#}$ and $F_{\varphi}$ are $\kappa$-basically disconnected.

Proof. Parts (1) and (2) follow directly from Lemma 1.1 and Theorem 5.1. Part 3 follows from Theorem 3.3: $F_{\varphi}$ is a retract of $F_{\varphi}{ }^{\#}$ and the results in part 2.

I was unable to answer the following question.

Question 5.3. Let $X$ be a compact $\kappa$-basically disconnected space. Do there exist selfmaps $\varphi$ on $X$ for which the set $F_{\varphi}{ }^{\#}$ is not a $P_{\kappa}$-set? Note that $F_{\varphi}$ is always basically disconnected. Is it always a $\kappa$-basically disconnected subspace? And is the set $F_{\varphi}$ a basically disconnected, respectively a $\kappa$-basically disconnected subspace, for all maps $\varphi$ ?

There exists a large class of nice maps to which Corollary 5.2 can be applied, the open maps. In particular, if $\varphi$ is an autohomeomorphism of a compact $\kappa$-basically disconnected space, then its fixed-point set is a $P_{\kappa}$-set.

Note that a map $\varphi$ on a compact $\kappa$-basically disconnected space $X$ need not be $\# \kappa$, not even if the map is an embedding. For example, in [Wal] an example is presented of a basically disconnected space together with an embedding $\varphi$ with the properties that its (unique) fixed point is not an attractor. It follows from Proposition 3.2 that this particular map cannot be a $\# \omega_{1}$-map. However, the following result tells us that for embeddings (in the class of compact $\kappa$-basically disconnected spaces) the set $F_{\varphi}$ is always a $P_{\kappa}$-set and therefore $\kappa$-basically disconnected.

Theorem 5.4. Let $X$ is a compact $\kappa$-basically disconnected space and $\varphi$ an embedding of $X$ into itself. Then the fixed point set $F_{\varphi}$ of $\varphi$ is a $P_{\kappa}$-set.

Moreover, if $X-F_{\varphi}$ is the union of at most $\kappa$ closed sets, then $X-F_{\varphi}$ is the union of three pairwise disjoint open sets $U^{i}$ with the property $\varphi\left(U^{i}\right) \cap U^{i}=\varnothing$, for all $i$.

Proof. This is proved by induction on $\kappa$.

It follows from Theorem 4.2 that is true for $\kappa=\omega_{1}$. Let $\kappa_{0}$ be an ordinal, and assume that the statement is true for all selfembeddings of all compact $\kappa$-basically disconnected spaces, with $\kappa<\kappa_{0}$.

Fix a selfembedding $\varphi$ of a compact $\kappa_{0}$ basically disconnected space $X$. By assumption, $F_{\varphi}$ is a $P_{\kappa}$-set, for all $\kappa<\kappa_{0}$. This already proves the claim, in the case $\kappa_{0}$ is a limit cardinal.

Assume $\kappa_{0}=\gamma^{+}$. Let $\left\{A_{\alpha}: \alpha<\gamma\right\}$ be a family of less than $\kappa_{0}=\gamma$ closed subsets disjoint from $F_{\varphi}$. The induction assumption gives us:

$$
\text { For all } \beta<\gamma: \operatorname{cl}\left[\bigcup\left\{A_{\alpha}: \alpha<\beta\right\}\right] \cap F_{\varphi}=\varnothing \text {. }
$$

Using this fact and the method in the proof of Theorem 5.1 one can easily construct (by induction ) a family $\left\{U_{\alpha}: \alpha<\gamma\right\}$ of clopen subsets satisfying the following properties:

(1) $A_{\alpha} \subset U_{\alpha}$.

(2) $U_{\alpha} \cap F_{\varphi}=\varnothing$.

(3) $\alpha<\beta$ implies $U_{\alpha} \subset U_{\beta}$.

(4) $\varphi\left(U_{\alpha}\right) \subset U_{\alpha}$. 
Note that $U=\bigcup\left\{U_{\alpha}: \alpha<\gamma\right\}$ is $C^{*}$-embedded. So, if it can be verified that $U$ has a 3-partition, then it follows that $\operatorname{cl}(U) \cap F_{\varphi}=\varnothing$. To obtain this 3partition of $U$ each set $U_{\alpha}$ will be 3-partitioned. By induction a family of 3-partitions $\left\{D_{\alpha}^{0}, D_{\alpha}^{1}, D_{\alpha}^{2}\right\}$ is defined, existing of sets clopen in $X$ satisfying:

(5) $\bigcup\left\{D_{\alpha}^{i}: i \in\{0,1,2\}\right\}=U_{\alpha}$.

(6) $D_{\alpha}^{i} \cap D_{\alpha}^{j}=\varnothing$, if $i \neq j$.

(7) $\varphi\left(D_{\alpha}^{i}\right) \cap D_{\alpha}^{i}=\varnothing$, for all $i$.

(8) $\alpha<\beta$ implies $D_{\beta}^{i} \cap U_{\alpha}=D_{\alpha}^{i}$.

Indeed, assume for $\alpha<\beta$ the partitions are defined.

Put $D_{i}=\bigcup\left\{D_{\alpha}^{i}: \alpha<\beta\right\}$. Then, $\left\{D_{0}, D_{1}, D_{2}\right\}$ is a partition of the $C^{*}$ embedded subset $U^{\beta}=\bigcup\left\{U_{\alpha}: \alpha<\beta\right\}$. Note that $\varphi\left(U^{\beta}\right) \subset U^{\beta}$ and $U^{\beta} \subset U_{\beta}$. The sets $D_{i}$ are relatively clopen in $U^{\beta}$ and $\varphi\left(D_{i}\right) \cap D_{i}=\varnothing$. It follows that $\Psi=\beta\left(\varphi \mid U^{\beta}\right)$ has the property: $\Psi\left(\operatorname{cl}_{\beta U^{\beta}}\left(D_{i}\right) \cap \mathrm{cl}_{\beta U^{\beta}}\left(D_{i}\right)=\varnothing\right.$. In particular, $\left\{\operatorname{cl} D_{0}, \operatorname{cl} D_{1}, \operatorname{cl} D_{2}\right\}$ is a 3-partition of the clopen set $\operatorname{cl} U^{\beta}$.

Finally use that the map $\varphi$ is injective, and conclude that Proposition 1.2 implies one can extend the 3-partition of $\mathrm{cl} U^{\beta}$ to a 3-partition of $U_{\beta}$.

Put $U_{i}=\bigcup\left\{D_{\alpha}^{i}: \alpha<\gamma\right\}$, then $\left\{U_{0}, U_{1}, U_{2}\right\}$ is the required 3-partition of $U$. The second claim can be proved in the same way as is done in Theorem 4.6

As an application we show the following:

Proposition 5.5. Let $X$ be a locally compact $\kappa$-basically disconnected spaces and assume that $X$ is the union of less than $\kappa$ compact subsets. If $\psi$ is a \#к-map without fixed points then $\beta \psi$ has no fixed points.

Moreover, if $\psi$ is an open map then $\beta \psi$ is an open map too.

Proof. The space $\beta X$ is compact $\kappa$-basically disconnected. Put $\varphi=\beta \psi$. The local compactness of $X$ obviously implies that $F_{\varphi}{ }^{\#} \subset \beta X-X$. Since $X$ is the union of less than $\kappa$ compact subsets, it follows that $\beta X-X$ does not contain nonempty sets which are $P_{\kappa}$-sets of $\beta X$. Therefore, if it can be verified that the map $\varphi: \beta X \rightarrow \beta X$ is $\# \kappa$, then it would follow that $F_{\varphi}^{\#}$, hence $F_{\varphi}$ is empty. Unfortunately, I cannot verify this. But what can be verified is that for every clopen set $C \subset \beta X$, the sequence

$$
C, C_{1}=C \cup \varphi^{-1} C, \ldots, C_{\omega_{0}}=\operatorname{cl}\left(\bigcup\left\{\varphi^{-k}(C)\right\}\right), \ldots
$$

will stabilize at ordinal level below $\kappa$. Whether this is equivalent with the property $\# \kappa$, I do not know. It is not difficult to verify that all the proofs of the

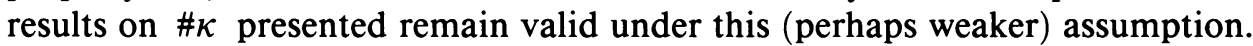
For the verification: let $C$ be clopen in $\beta X$. Consider the set $C^{\prime}=C \cap X$ and the sequence:

$$
C^{\prime}, C_{1}^{\prime}=C \cup \psi^{-1} C^{\prime}, \ldots, C_{\omega_{0}}^{\prime}=\operatorname{cl}\left(\bigcup\left\{\psi^{-k}(C)\right\}\right), \ldots
$$

This sequence stabilizes on an ordinal level $\alpha$ with $\alpha<\kappa$, and a clopen set $D$ is obtained with $C^{\prime} \subset D \subset X$ and with the property that $X-D$ is $\psi$-invariant. It follows that $\operatorname{cl}_{\beta X}(D)$ is clopen in $\beta X$ and also that $\beta X-\operatorname{cl}_{\beta X}(D)$ is $\varphi$ invariant. This implies that $C^{\#} \subset \operatorname{cl}_{\beta X} D$, and therefore the set $C^{\#}$ is obtained at most at ordinal level $\alpha+\omega_{0}$, which is below $\kappa$. 
Next, assume that the map $\varphi$ is open. Let $C$ be a clopen subset of $\beta X$. Again consider $C^{\prime}=C \cap X . X$ is locally compact, so: $C^{\prime}=\bigcup\left\{K_{\alpha}: \alpha<\gamma\right\}$, with $K_{\alpha}$ open and compact and $\gamma<\kappa$.

It follows that each $K_{\alpha}$ and each $\varphi\left(K_{\alpha}\right)$ is clopen in $\beta X$ and clearly the set $\beta \varphi(C)=\sup \left\{\varphi\left(K_{\alpha}\right): \alpha<\gamma\right\}$ is the supremum of less than $\kappa$ clopen sets, therefore clopen. It follows that $\beta \varphi$ is open.

\section{Extremally DisconNeCted SPACes.}

As a corollary of the results on $\kappa$-basically disconnected spaces, the following results are obtained as limit cases. Indeed, any map on a extremally disconnected space is \# $\kappa$ for some $\kappa$. The results obtained on the class of compact $\kappa$-basically disconnected spaces are already strong enough to reobtain some known theorems. For example, from Theorem 5.1 and Theorem 5.4 follows:

Theorem 6.1. Let $X$ be a a compact extremally disconnected space and let $\varphi$ be a map.

(1) $[\mathrm{A}, \mathrm{A}, \mathrm{K}]$ The set $F_{\varphi}{ }^{\#}$ is clopen.

(2) [F] If $\varphi$ is an embedding then $F_{\varphi}$ is clopen.

Corollary 6.2. Let $X$ be a compact extremally disconnected space and let $\varphi$ be a map. Then $X$ can be written as the disjoint union of four clopen subsets $C_{i}$, $(i=1,2,3,4)$ with the following properties:

(1) $C_{i} \cap C_{j}=\varnothing$, if $i \neq j$.

(2) $C_{1} \cup C_{2} \cup C_{3} \cup C_{4}=X$.

(3) $\varphi\left(C_{1} \cup C_{2} \cup C_{3}\right) \subset C_{1} \cup C_{2} \cup C_{3}$.

(4) $\varphi\left(C_{4}\right) \subset C_{4}$ and $\varphi\left(C_{i}\right) \cap C_{i}=\varnothing$, for $1=1,2,3$.

(5) If $U$ is (cl)open with $\varphi(U) \subset U$ then: $U$ contains a fixed point if and only if $U \cap C_{4} \neq \varnothing$.

Moreover, in this decomposition the set $C_{4}$ is uniquely determined.

Proof. Of course, we take $C_{4}=F_{\varphi}{ }^{\#}$ and as $\left\{C_{1}, C_{2}, C_{3}\right\}$ one can take just any 3-partition of the clopen set $X-F_{\varphi}{ }^{\#}$. For the uniqueness of $C_{4}$ we observe that the properties of $C_{4}$ implies the following:

(1) $F_{\varphi} \subset C_{4}$.

(2) $F_{\varphi}{ }^{\#} \subset C_{4}$, as the complement of $C_{4}$ is $\varphi$-invariant.

We have seen in previous proofs that every $x \in X-F_{\varphi}{ }^{\#}$ is contained in a cozeroset $U$ with $\varphi(U) \subset U$ and $x \in U \subset X-F_{\varphi}{ }^{\#}$. It follows that $U \cap C_{4}=$ $\varnothing$, and so $C_{4}=F_{\varphi}{ }^{\#}$.

Another result is that in the class of compact extremally disconnected spaces the class of fixed-points subsets is as small as possible. Precisely the retracts! Not only this, the discussion at the end of section 3 shows that in some sense every continuous selfmap on a compact extremally disconnected space already carries the structure of the retraction of its fixed-point set on the clopen set $F_{\varphi}{ }^{\#}$. We collect some properties of fixed-points sets in one theorem.

Theorem 6.3. Let $X$ be a a compact extremally disconnected space and let $\varphi$ be a map. Then 
(1) If $F_{\varphi} \neq \varnothing$ then $F_{\varphi}$ is a retract of $X$ and hence extremally disconnected.

(2) $d\left(F_{\varphi}\right) \leq d(X)$ and $c\left(F_{\varphi}\right) \leq c(X)$.

(3) $X-F_{\varphi}$ is pseudocompact.

Proof. The first two statements are clear. Probably the third statement is wellknown but I do not have the proper references. The complement of a compact extremally disconnected subspace is always pseudocompact, for assume $X-F_{\varphi}$ is not pseudocompact. Choose a $C$-embedded (closed) copy $\left\{x_{n}: n \in \omega_{0}\right\}$ of $\mathbb{N}$ in $X-F_{\varphi}$. Find pairwise disjoint clopen sets $C_{n}$ with:

- $x_{n} \in C_{n}$.

- $C_{n} \cap F_{\varphi}=\varnothing$.

Define $r: \cup C_{n} \rightarrow\left\{x_{n}: n \in \omega_{0}\right\}$ by $r\left(C_{n}\right)=x_{n}$. This map $r$ can be extended to a retraction

$$
\beta r: \operatorname{cl}\left[\bigcup C_{n}\right] \rightarrow \operatorname{cl}\left[\left\{x_{n}: n \in \omega_{0}\right\}\right]
$$

The set $\operatorname{cl}\left[\bigcup C_{n}\right]$ is clopen, and will intersect $F_{\varphi}$. Indeed,

$$
\operatorname{cl}\left\{x_{n}: n \in \omega_{0}\right\}-\left\{x_{n}: n \in \omega_{0}\right\} \subset \operatorname{cl}\left[\bigcup C_{n}\right] \cap F_{\varphi} .
$$

The clopen subset $\operatorname{cl}\left[\cup C_{n}\right] \cap F_{\varphi}$ of $F_{\varphi}$ has a retraction onto the space $\omega^{*}$, which is not extremally disconnected, and therefore the clopen subspace and $F_{\varphi}$ itself cannot be extremally disconnected.

Another perhaps surprising result is the following result.

Theorem 6.4. Let $X$ be a locally compact extremally disconnected space and let $\varphi$ be a map. Then:

(1) The following are equivalent:

(a) $\varphi$ has no fixed points.

(b) $\beta \varphi$ has no fixed points.

(2) $\operatorname{cl}_{\beta X}\left(F_{\varphi}\right)=F_{\beta \varphi}$.

(3) $F_{\varphi}$ is extremally disconnected.

Proof. The sharp operator \# will denote the one on $\beta X$.

(1) If $F_{\beta \varphi} \neq \varnothing$ then, being clopen, $F_{\beta \varphi}^{\#} \cap X \neq \varnothing$.

But $X$ is open in $\beta X$, so Lemma 4.1 implies that $X \cap F_{\beta \varphi} \neq \varnothing$. This gives that $\varphi$ has a fixed point. The other direction is trivial.

(2) The inclusion $\operatorname{cl}_{\beta X}\left(F_{\varphi}\right) \subset F_{\beta \varphi}$ is trivial.

To obtain the other inclusion, let us assume that $\operatorname{cl}_{\beta X}\left(F_{\varphi}\right)$ is a proper subset of $F_{\beta \varphi}$. Choose $t \in F_{\beta \varphi}-\operatorname{cl}_{\beta X}\left(F_{\varphi}\right)$. Then there exists a set $C$ with:

(1) $C$ (relatively) clopen in $F_{\beta \varphi}$.

(2) $\operatorname{cl}_{\beta X}\left(F_{\varphi}\right) \subset C \subset F_{\beta \varphi}-\{t\}$.

It follows from Theorem 3.3 that the set $D=F_{\beta \varphi}{ }^{\#}-C^{\#}$ is clopen and $\beta \varphi(D) \subset$ $D$. The subspace $D \cap X$ is locally compact and extremally disconnected, and $\varphi(D \cap X) \subset D \cap X$. Clearly the map $\varphi \mid D$ is a map without fixed points with the property that $\beta(\varphi \mid D)$ has a fixed point, namely $t$. This contradiction proves the claim.

(3) It follows from Theorem 6.3 that the space $F_{\beta \varphi}$ is extremally disconnected. But then, the set $F_{\varphi}$, which is dense in $F_{\beta \varphi}$, is extremally disconnected too. 
Question 6.5. Does there exists an extremally disconnected space $Y$ with a fixedpoint free map $\varphi: Y \rightarrow Y$ such that $\beta \varphi$ does have fixed points?

Corollary 6.6. Let $X$ be a locally compact extremally disconnected space and let $\varphi$ be a map. Then $X$ can be written as the disjoint union of four clopen subsets $C_{i},(i=1,2,3,4)$ with the following properties:

(1) $C_{i} \cap C_{j}=\varnothing$, if $i \neq j$.

(2) $C_{1} \cup C_{2} \cup C_{3} \cup C_{4}=X$.

(3) $\varphi\left(C_{1} \cup C_{2} \cup C_{3}\right) \subset C_{1} \cup C_{2} \cup C_{3}$.

(4) $\varphi\left(C_{4}\right) \subset C_{4}$ and $\varphi\left(C_{i}\right) \cap C_{i}=\varnothing$, for $1=1,2,3$.

(5) If $U$ is (cl)open with $\varphi(U) \subset U$ then: $U$ contains a fixed point if and only if $U \cap C_{4} \neq \varnothing$.

Moreover, in this decomposition the set $C_{4}$ is uniquely determined.

Proof. Restrict the partition described in Corollary 6.2 to $X$.

Lately several results have appeared (e.g. [K,S], [vD]) in the area concerning types of maps and spaces that satisfy the fixed point free extension property. As an application of the results presented, the following fits in this area of results.

Proposition 6.7. Let $Y$ be a locally compact space and let $\varphi$ be a map on $Y$ without fixed points. If $x \in \beta Y$ is a fixed point of $\beta \varphi$ then the space $\beta Y$ is not extremally disconnected at $x$. In particular, $x$ is not a remote point of $\beta Y$.

Proof. Consider the Gleason cover of the spaces $Y$ and $\beta Y$, together with the maps $\pi: E Y \rightarrow Y$ and $\pi^{\beta}: E \beta Y \rightarrow \beta Y$. The space $E Y$ is considered to be a dense subspace of $E \beta Y$ and $\pi^{\beta} \mid E Y=\pi$.

Recall the following basic properties of the Gleason cover $\pi: E X \rightarrow X$ of a space $X$ :

(1) $E X$ is extremally disconnected.

(2) $\beta E X=E \beta X$ and $\beta \pi=\pi^{\beta}$.

(3) Every map $f: X \rightarrow X$ can be lifted to a map $E f: E X \rightarrow E X$ with the property: $f \circ \pi=\pi \circ E f$. Such a map is called a lifting of $f$. A lifting is not unique, unless the map $f$ is open.

(4) If $p \in \beta X-X$ then the following are equivalent:

- $\beta X$ is extremally disconnected in $p$.

- If $U$ and $V$ are disjoint open sets of $X$, then $p \notin \operatorname{cl}_{\beta X}(U) \cap$ $\mathrm{cl}_{\beta X}(V)$.

- card $\pi^{-1}\{p\}=1$.

Consider the fixed point free map $\varphi: Y \rightarrow Y$, together with a lifting $E \varphi$ : $E Y \rightarrow E Y$. The map $E \varphi$ has no fixed point either and it follows that the map $\beta E \varphi$ cannot have fixed points. (The space $E Y$ is extremally disconnected and locally compact.)

Note also that the map $\beta E \varphi$ can be considered to be a lifting of the map $\beta \varphi$. Therefore, if $y \in \beta Y-Y$ and $z \in\left(\pi^{\beta}\right)^{-1}(y)$ then $\beta E \varphi(z) \in\left(\pi^{\beta}\right)^{-1}(y)$.

In particular, if $y$ is a fixed point of $\beta \varphi$ and $\beta Y$ is extremally disconnected at $y$ (say $y^{\prime}$ is the unique point in $\left(\pi^{\beta}\right)^{-1}(y)$ ), then $\beta E \varphi\left(y^{\prime}\right)=y^{\prime}$, so the map $\beta E \varphi$ would have a fixed point after all. Contradiction. 


\section{PeRiodic POINTS OF MAPS ON EXTREMALly DisCONNECTED SPACES}

Let $X$ be a compact extremally disconnected space and let $\varphi$ be a continuous map on $X$. For each $n$ define

$$
\begin{gathered}
P^{n}=\left\{x \in X: \varphi^{n}(x)=x \text { and } \varphi^{m}(x) \neq x, \text { for } m<n\right\}, \\
P_{\varphi}=\bigcup\left\{P^{n}: n \geq 1\right\} .
\end{gathered}
$$

The points in $P_{\varphi}$ are called the periodic points, a point in $P^{n}$ is a point of period $n$. Clearly, $P^{1}=F_{\varphi}$ and $\bigcup\left\{P^{i}: i\right.$ divides $\left.n\right\}=F_{\varphi^{n}}$.

In general, the sets $P^{n}$ need not be closed, as easy examples show. However, if the space $X$ is compact extremally disconnected we will see that these sets are closed.

Lemma 7.1. Let $X$ be a compact extremally disconnected space and let $\varphi$ be a continuous map on $X$. Then $F_{\varphi}{ }^{\#} \cap P_{\varphi}=F_{\varphi}$.

In particular, $P^{2}$ is a closed set.

Proof. Assume $p \in F_{\varphi}{ }^{\#}$ with $\varphi^{n}(p)=p, n>1$ and $p \notin F_{\varphi}$. Since $p$ is a fixed point of $\varphi^{n}$, and therefore a strong attractor, there exists a clopen set $W$ with $p \in W, W \cap F_{\varphi}=\varnothing$ and $\varphi^{n}(W) \subset W$. Since $W \cap F_{\varphi}{ }^{\#} \neq \varnothing$, the ordinal $\alpha=\min \left\{\beta: W \cap\left(F_{\varphi}\right)_{\beta} \neq \varnothing\right.$ is well defined. The same argument as in Lemma 4.1 shows that $\alpha=0$. Contradiction. It follows that $P^{2}=F_{\varphi^{2}}-F_{\varphi}{ }^{\#}$ and as the set $F_{\varphi}{ }^{\#}$ is clopen we see that $P^{2}$ is a closed subset.

Notation: If $X$ is a space and two maps $\varphi, \psi$ are given on $X$ then, for any $A \subset X$ the sets $A_{\varphi}^{\#}$ and $A_{\psi}^{\#}$ denotes the sharps of this set with respect to these two maps.

Lemma 7.2. Let $X$ be a compact extremally disconnected space and let $\varphi$ be a continuous map on $X$. Consider the maps $\varphi, \varphi^{2}: X-F_{\varphi}^{\#} \rightarrow X-F_{\varphi}{ }^{\#}$ on $X-F_{\varphi}^{\#}$. Then:

(1) $P^{2} \subset X-F_{\varphi}^{\#}$.

(2) $P_{\varphi}^{2}{ }^{\#}=P_{\varphi^{2}}^{2}$.

(3) $P_{\varphi} \cap P_{\varphi}^{2}{ }^{\#}=P^{2}$.

(4) There exists a retraction $r_{2}: P_{\varphi^{2}}^{2} \rightarrow P^{2}$ such that $r_{2} \circ \varphi^{2}=r_{2}$.

Proof. (1) This is clear from the previous lemma.

(2) The inclusion: $P_{\varphi^{2}}^{2} \subset P_{\varphi}^{2}{ }^{\#}$ is clear, as the second set is $\varphi$-invariant, therefore $\varphi^{2}$-invariant closed and contains $P^{2}$. It is clear that $\varphi\left(P^{2}\right)=P^{2}$ and therefore

$$
\bigcup\left\{\varphi^{-k}\left(P^{2}\right): k \in \omega_{0}\right\}=\bigcup\left\{\left(\varphi^{2}\right)^{-k}\left(P^{2}\right): k \in \omega_{0}\right\} .
$$

In particular,

$$
\varphi\left(\operatorname{cl}\left[\bigcup\left\{\left(\varphi^{2}\right)^{-k}\left(P^{2}\right): k \in \omega_{0}\right\}\right]\right) \subset \operatorname{cl}\left[\bigcup\left\{\left(\varphi^{2}\right)^{-k}\left(P^{2}\right): k \in \omega_{0}\right\}\right]
$$

It follows that on the limit levels, in the inductive construction of $P_{\varphi^{2}}^{\#}$, there will always appear sets which are $\varphi$-invariant. In particular, $P_{\varphi^{2}}^{\#}$ is $\varphi$-invariant, and the conclusion follows. 
(3) Note that if we put $Y=X-F_{\varphi}{ }^{\#}$ and $\psi=(\varphi \mid Y)^{2}$, then $Y$ is compact extremally disconnected and $P^{2}=F_{\psi}$. It follows from the first lemma that $F_{\psi}{ }^{\#}-F_{\psi}$ contains no periodic points of $\psi$, and therefore not of $\varphi$. And the previous result gives the conclusion.

(4) This is clear.

Question 7.3. (1) Does there also exist a retraction $r_{2}: P_{\varphi^{2}}^{2} \rightarrow P^{2}$ such that $r_{2} \circ \varphi=r_{2}$ ?

(2) Let $X$ be a compact $\kappa$-basically disconnected space and let $\varphi$ be open, $\# \kappa$ or an embedding. Is it still true that the sets $P^{n}$ are closed?

The argument in the previous lemma can be used for all $n$, and we conclude that for maps $\varphi$ on a compact extremally disconnected space $X$ :

(1) Each $P^{n}$ is a closed subset.

(2) For all $n$ : consider the maps $\varphi$ and $\varphi^{n}$ on the subspace $X-\bigcup\left\{\left(P^{i}\right)^{\#}\right.$ : $i \in n\}$ of $X$.

Then:

- $P^{n} \subset X-\bigcup\left\{\left(P^{i}\right)^{\#}: i \in n\right\}$.

- $P_{\varphi}^{n}{ }_{\varphi}^{\#}=P_{\varphi^{n}}^{\#}$.

So, the notation $\left(P^{n}\right)^{\#}$ is unambiguous.

(3) $P_{\varphi} \cap\left(P^{n}\right)^{\#}=P^{n}$.

(4) The family $\left\{P^{n}: n \in \omega_{0}\right\}$ is a discrete family, in the sense that the members are contained in pairwise disjoint clopen sets, namely:

(5) The family $\left\{\left(P^{n}\right)^{\#}: n \in \omega_{0}\right\}$ is a pairwise disjoint family of clopen sets.

(6) For each $n$ there exists a retraction $r_{n}:\left(P^{n}\right)^{\#} \rightarrow P_{n}$ such that $r_{n}=$ $r_{n} \circ \varphi^{n}$.

Corollary 7.4. Let $X$ be a compact extremally disconnected space and let $\varphi$ be a continuous map. Then:

(1) The subspace $\operatorname{cl}\left(\bigcup\left\{P^{n}: n \in \omega_{0}\right\}\right)$ is a retract of $X$. In particular, the subspace $\operatorname{cl}\left(\bigcup\left\{P^{n}: n \in \omega_{0}\right\}\right)$ is extremally disconnected.

(2) If the map is open then: $\operatorname{cl}\left[\bigcup\left\{\left(P^{n}\right)^{\#}: n \in \omega_{0}\right\}\right]=\left(\operatorname{cl}\left[\bigcup\left\{P^{n}: n \in \omega_{0}\right\}\right]\right)^{\#}$. Proof. Consider the retractions $r^{n}:\left(P^{n}\right)^{\#} \rightarrow P^{n}$. Since the members in $\left\{\left(P^{n}\right)^{\#}: n \in \omega_{0}\right\}$ are pairwise disjoint and clopen, we see that the retraction:

$$
r=\bigoplus r^{n}: \bigoplus\left(P^{n}\right)^{\#} \rightarrow \bigoplus P^{n}
$$

can be extended to a retraction

$$
\beta r=\beta \oplus r^{n}: \beta\left(\bigoplus\left(P^{n}\right)^{\#}\right) \rightarrow \beta\left(\bigoplus P^{n}\right) .
$$

But $\beta\left(\bigoplus\left(P^{n}\right)^{\#}\right)=\operatorname{cl}\left[\bigcup\left\{P^{n}\right)^{\#}: n \in \omega_{0}\right\}$ and $\beta\left(\oplus P^{n}\right)=\operatorname{cl} \bigcup\left\{P^{n}: n \in\right.$ $\left.\left.\omega_{0}\right)\right\}$. The conclusion follows. The second statement can be proved as in Lemma 2.2.

\section{WHEN ARE FIXED POINT SETS CLOPEN?}

We have obtained results that explain why the fixed-point set of an embedding of an extremally disconnected space is clopen. In this section we want to look 
for internal properties that also give this information. What is meant by this is shown by the following proposition.

Proposition 8.1. Let $X$ be a compact extremally disconnected space and let $\varphi$ be a continuous map. The following are equivalent:

(1) $F_{\varphi}$ is clopen in $X$.

(2) $F_{\varphi}$ is clopen in $F_{\varphi}{ }^{\#}$.

(3) $F_{\varphi}$ is (relatively) open in $\varphi^{-1}\left(F_{\varphi}\right)$.

Proof. Most of the implications are trivial. The equivalence of 1 and 2 is clear from Theorem 6.1. The only non-trivial implication one is $3 \rightarrow 2$. If $F_{\varphi}$ is clopen in $\varphi^{-1}\left(F_{\varphi}\right)$, then there exists a clopen (in $\left.X\right)$ set $C$ with $\varphi^{-1}\left(F_{\varphi}\right) \subset$ $C \subset X-F_{\varphi}$. From Lemma 3.1 we conclude that: $F_{\varphi} \cap C^{\#}=\varnothing$. Since $\varphi^{-1}\left(F_{\varphi}\right)-F_{\varphi} \subset C$, the inductive construction and the previous formula implies that: $F_{\varphi}^{\#}-C^{\#}=F_{\varphi}$. As $C^{\#}$ is clopen in $X$, the conclusion follows. Note that the statements 2 and 3 are equivalent in the class of compact $\kappa$-basically disconnected spaces and the \# $\kappa$-maps.

So, for compact extremally disconnected spaces, the retraction

$$
\varphi \mid \varphi^{-1}\left(F_{\varphi}\right): \varphi^{-1}\left(F_{\varphi}\right) \rightarrow F_{\varphi}
$$

decides whether $F_{\varphi}$ is clopen in $X$. For categorical reasons, the following question becomes interesting.

Question 8.2. Let $X$ be a compact extremally disconnected space and let $\varphi$ be a continuous map. Is the subspace $\varphi^{-1}\left(F_{\varphi}\right)$ always extremally disconnected? Is it always a retract?

The following statement follows straightforward by from the fact that $F_{\varphi}{ }^{\#}$ is clopen. It is also a corollary of Proposition 8.1.

Corollary 8.3. Let $X$ be a compact extremally disconnected space and let $\varphi$ be a continuous map. If, for all $x \in F_{\varphi}$, card $\varphi^{-1}\{x\}=1$, then the set $F_{\varphi}$ is clopen.

In $[\mathrm{A}, \mathrm{A}, \mathrm{K}]$ it was proved that every map $\varphi$ of finite order on a compact extremally disconnected space $X$, has a clopen fixed point set. Comparing this with Proposition 8.1 the following question appears rather natural.

Question 8.4. Let $X$ be a compact extremally disconnected space and let $\varphi$ be a continuous map. Assume there exists $k$ such that card $\varphi^{-1}\{x\} \leq k$, for all $x \in F_{\varphi}$. Does this imply that the fuxed point set $F_{\varphi}$ is clopen?

The following question was posed in $[\mathrm{A}, \mathrm{A}, \mathrm{K}]$, and remains unanswered.

Question 8.5. Let $X$ be a compact extremally disconnected space and let $\varphi$ be an open continuous map. Is the fixed point set necessarily clopen?

Note that this question is equivalent with the question whether the retraction $r: F_{\varphi}{ }^{\#} \rightarrow F_{\varphi}{ }^{\#}$, as defined in section 2 , is open. In this connection the following observation might be interesting.

Lemma 8.6. Let $X$ be a compact extremally disconnected space and let $\varphi$ be an open continuous map. The retraction $r: F_{\varphi}{ }^{\#} \rightarrow F_{\varphi}$ is an open map. 
Proof. Let $C$ be a clopen set in $F_{\varphi}{ }^{\#}$. It is easy to check that

$$
r(C)=\operatorname{cl}\left[\bigcup\left\{\varphi^{n}(C) \cap F_{\varphi}: n \in \omega_{0}\right\}\right] .
$$

The second set is open in $F_{\varphi}$, since:

(1) All the sets $\varphi^{n}(C) \cap F_{\varphi}$ are open in $F_{\varphi}$.

(2) $F_{\varphi}$ is extremally disconnected.

The conclusion follows.

If the answer to the Question 8.5 is no, the following interesting example appears. Let $Y$ be a compact extremally disconnected space an open continuous map $\psi: Y \rightarrow Y$, whose fixed point set $F_{\psi}$ is not clopen.

Consider the closed set: $A=F_{\psi}-$ int $_{X} F_{\psi}$. This set is clopen in $F_{\psi}$, and therefore:

There will exist an extremally disconnected space $X$ with an open continuous map $\varphi$ with non-empty nowhere dense fixed point set.

Indeed, take $X=A^{\#}$ and $\varphi=\psi \mid A^{\#}$.

As the map $\varphi$ is open, all the sets $\varphi^{-n}\left(F_{\varphi}\right)$ are nowhere dense. Note that $\cup \varphi^{-n}\left(F_{\varphi}\right)$ is dense in $X$ (see Lemma 2.2) and that Baire's theorem implies that the subspace $E=X-\bigcup \varphi^{-n}\left(F_{\varphi}\right)$ and therefore: $E$ is extremally disconnected and $\beta E=X$.

It follows that: there exist a Čech-complete extremally disconnected space $E$ and an open continuos map $(=\varphi \mid E)$ without fixed points whose Čech-Stone extension has fixed points. Compare this with Theorem 6.4.

Note that for every map $\varphi$ on a space $X$ there exist open subsets $U$ which are maximal with respect to the properties of being open with $\varphi(U) \cap U=\varnothing$. Such a set is called $\varphi$-disjoint.

One of the reasons that open maps $\varphi$ on compact extremally disconnected spaces might have clopen fixed point sets is the fact that in such a case such a maximal open $\varphi$-disjoint set is neccesairly clopen.

Moreover, it is easy to see that the fixed-point set $F_{\varphi}$ is clopen if and only if $X-F_{\varphi}$ is the union of three (finitely many) clopen sets $C_{i}$ with $\varphi\left(C_{i}\right) \cap C_{i}=\varnothing$.

Lemma 8.7. Let $X$ be a compact extremally disconnected space and let $\varphi$ be an open continuous map. If $C$ is a maximal $\varphi$-disjoint clopen set, then

(i) $\varphi^{-1}\left(F_{\varphi}\right)-F_{\varphi} \subset C \cup \varphi(C)$.

(ii) $F_{\varphi}^{\#}-F_{\varphi} \subset C^{\#} \cup \varphi(C)$.

Proof. Assume this is not the case. In the first case one can conclude that there exists $x \in X$ with $x \in \varphi^{-1}\left(F_{\varphi}\right)-F_{\varphi}$ and with $x \notin C \cup \varphi(C)$. Then $\varphi(x) \in F_{\varphi}$, so $\varphi(x) \notin C$. In the second case there exists $x$ with $x \notin F_{\varphi}$ and $x \notin C{ }^{\#} \cup \varphi(C)$. But then $\varphi(x) \notin C$ (as $x \notin C{ }^{\#}$ ). In either case there exists $x \in X=F_{\varphi}^{\#}$ with $x \notin C \cup \varphi(C), \varphi(x) \notin C$ and $\varphi(x) \neq x$.

Choose clopen sets $U$ and $V$ with

$$
\begin{gathered}
x \in U, \varphi(x) \in V, \varphi(U) \subset V, U \cap V=\varnothing, \\
U \cap(C \cup \varphi(C))=\varnothing, V \cap C=\varnothing .
\end{gathered}
$$


It is easy to verify that

$$
(C \cup U) \cap \varphi(C \cup U)=\varnothing
$$

contradicting the maximality of $C$.

This lemma enables us to answer Question 8.4 for the the class of open maps.

Proposition 8.8. Let $X$ be a compact extremally disconnected space and let $\varphi$ be an open continuous map. The following are equivalent for (a fixed) $x_{0} \in F_{\varphi}$.

(1) $x_{0} \in$ int $_{X} F_{\varphi}$.

(2) $x_{0} \notin \operatorname{cl}\left[\varphi^{-1}\left\{x_{0}\right\}-\left\{x_{0}\right\}\right]$.

Proof. We may assume that $X=F_{\varphi}$. For the proof $(2) \rightarrow(1)$ we separate the following cases.

Case 1. card $\varphi^{-1}\left\{x_{0}\right\}=1$.

Let $C$ be a maximal $\varphi$-disjoint clopen set. The previous lemma implies that $x_{0} \in F_{\varphi}^{\#}-\left(C C^{\#} \cup \varphi(C)\right) \subset F_{\varphi}$ and so $x_{0} \in \operatorname{int}\left(F_{\varphi}\right)$.

Case 2. The general case. Assume $x_{0} \notin \operatorname{cl}\left[\varphi^{-1}\left\{x_{0}\right\}-\left\{x_{0}\right\}\right]$. Choose a clopen set $C$ with $\operatorname{cl}\left[\varphi^{-1}\left\{x_{0}\right\}\right] \subset C \subset X-F_{\varphi}$. Since $C^{\#} \cap F_{\varphi}=\varnothing$ and $\varphi\left(X-C^{\#}\right) \subset X-C^{\#}$, it follows that the restriction of the map $\varphi$ to $X-C^{\#}$ translates the situation into Case 1 .

The conclusion follows.

Corollary 8.9. Let $X$ be a compact extremally disconnected space and let $\varphi$ be an open continuous map.

If the set $F=\left\{x \in F_{\varphi}: \varphi^{-1}\{x\}\right.$ is finite $\}$ is dense in $F_{\varphi}$, then $F_{\varphi}$ is clopen.

Remark. Recently E. Thummel constructed an example of an open map on a compact extremally disconnected space with non-clopen fixed-point set.

\section{REFERENCES}

[A,A,K] Y.A. Abramovich, E.L. Arenson and A.K. Kitover, Banach $C(K)$-modules and operator preserving disjointness, Pitman Res. Notes in Math. Ser., no. 277, Essex, England.

[B,F] B. Balcar and F. Franek, Universal minimal dynamical systems for discrete semigroups, manuscript.

[B,K] A. Błaszczyk and Kim Do Young, A topological version of a combinatorial theorem of Katětov, Comment. Math. Univ. Carolin. 29 (1988), 657-663.

[D] E. van Douwen, $\beta X$ and fixed-point free maps, Topology Appl. 51 (1993), 191-195.

[D,M] E.K. van Douwen and J. van Mill, Subspaces of basically disconnected spaces or quotients of countably complete Boolean algebras, Trans. Amer. Math. Soc. 259 (1980), 121-127.

[F] Z. Frolik, Fixed points of maps of extremally disconnected spaces and complete Boolean algebras, Bull. Acad. Polon. Sci. Sér. Sci. Math. Astronom. Phys. 16 (1968), 269-275.

[H,V] K.P. Hart and J. Vermeer, Homeomorphisms on F-spaces (to appear).

$\left[\mathrm{K}_{1}\right] \quad$ A.K. Kitover, Operators preserving disjointness and the mappings of extremally disconnected compact spaces, Optimizatsiya 40 (1987), 138-143.

[K $\left.\mathrm{K}_{2}\right]$ Kim Do Young, Ph.D. thesis.

[K,S] A. Krawczyk and J. Steprāns, Continuous colourings of closed graphs, Topology Appl. 51 (1993), 13-27. 
[vM] J. van Mill, An introduction to $\beta \omega$, Handbook of Set-Theoretical Topology, NorthHolland, 1984.

[ $\left.\mathrm{V}_{1}\right] \quad \mathrm{J}$. Vermeer, Continuous maps on finite dimensional spaces (manuscript).

[ $\left.\mathrm{V}_{2}\right] \quad$, Frolik's theorem for basically disconnected spaces, Acta Univ. Carolin. 34 (1993), 135-142.

[Wal] R.C. Walker, The Stone-Čech compactification, Springer-Verlag, 1974.

Faculty of Technical Mathematics and Informatics, TU Delft, Postbus 5031, 2600 Ga Delft, The Netherlands

E-mail address: J.Vermeer@twi.tudelft.nl 